\title{
Interactive comment on "Data assimilation as a deep learning tool to infer ODE representations of dynamical models” by Marc Bocquet et al.
}

\section{Marc Bocquet et al.}

marc.bocquet@enpc.fr

Received and published: 28 May 2019

May 28, 2019

Subject: Manuscript npg-2019-7: "Data assimilation as a deep learning tool to infer ODE representations of dynamical models", Response to Reviewer 2. 
account to improve the manuscript. We respond below to these comments and tell how we modified the manuscript accordingly. A pdf file showing the differences between the NPGD original and the revised manuscript is provided.

1. p.6, lines 15-18. The locality assumption is obviously helpful since it reduces number of regressors, but I wouldn't go as far as claiming that long-range dependencies are precluded in geophysical applications. For example long-range teleconnections in coupled ocean-atmosphere system are well established.

As a matter of fact, we were pointing to the absence of long-range instantaneous interactions which are indeed precluded in geophysical fluids. This does not prevent the building up of teleconnections and specific long-range dependencies. Following your comment, we have added the sentence: "This would not prevent potential specific long-distance dependencies (such as teleconnections)."

2. Section 2.2.2. The homogeneity assumption is also useful since it simplifies even more estimation of the surrogate model and obviously helps with robustness. On the other hand, the toy-model examples largely favor this assumption in a sense that the statistical properties of state variables are basically the same, namely $x$ variables in L96, L05III and KS. However in real-world applications this is hardly the case, for example ENSO dynamics of sea surface temperatures in tropical Pacific which is very inhomogeneous. It would be helpful to have authors elaborate more on this point, i.e. in lines 23-25, $p 6$.

Yes, we agree with you. We have further developed the paragraph by adding: "For realistic geofluids, the forcing fields (solar irradiance, bathymetry, boundary conditions, friction, etc.) are heterogeneous, so that the homogeneity assumption should be dropped. Nonetheless, the fluid dynamics part of the model would remain homogeneous. As a result, an hybrid approach could be enforced." Thank you for the suggestion.

Interactive

comment

Printer-friendly version

Discussion paper 
3. Section 3.1. Having monomials in the surrogate model can bring numerical instabilities, so it is nice to see that authors have plans to deal with this issue - (Eq.43) and NPGD lines 10-14 on p.14. However it is not clear if this remedy has been applied for the examples presented.

Yes, these techniques are systematically enforced in the numerical experiments. They are especially useful for large windows. In practice, they only operate in the first few iterations of the minimisation. Later, a stable integration of the model is reached and the rectifier functions $\zeta$ act linearly. The text has been slightly improve to reflect this.

4. The term "resolvent" was used multiple times, the meaning of it was not clear to me.

The resolvent is the model integrated in time, say from $t_{k}$ to $t_{k+1}$, as opposed to the flow rate, which is instantaneous and not integrated. The term is now defined when first appearing.

Marc Bocquet, Julien Brajard, Alberto Carrassi, Laurent Bertino.

Interactive comment on Nonlin. Processes Geophys. Discuss., https://doi.org/10.5194/npg2019-7, 2019. 